\title{
Update Breast Cancer 2017 - Implementation of Novel Therapies
}

\author{
Update Mammakarzinom 2017 - \\ Etablierung neuer zielgerichteter Therapien
}

Authors

Michael P. Lux ${ }^{1}$, Wolfgang Janni ${ }^{2}$, Andreas D. Hartkopf ${ }^{3}$, Naiba Nabieva ${ }^{1}$, Florin-Andrei Taran ${ }^{3}$, Friedrich Overkamp ${ }^{4}$, Hans-Christian Kolberg ${ }^{5}$, Peyman Hadji ${ }^{6}$, Hans Tesch ${ }^{7}$, Johannes Ettl ${ }^{8}$, Jens B. Huober ${ }^{2}$, Diana Lüftner ${ }^{9}$, Markus Wallwiener ${ }^{10}$, Volkmar Müller ${ }^{11}$, Matthias W. Beckmann ${ }^{1}$, Erik Belleville ${ }^{12}$, Tanja N. Fehm ${ }^{13}$, Diethelm Wallwiener ${ }^{3}$, Sara Y. Brucker ${ }^{3}$, Andreas Schneeweiss ${ }^{10,14}$, Peter A. Fasching ${ }^{1}$

Affiliations

1 Erlangen University Hospital, Department of Gynecology and Obstetrics, Comprehensive Cancer Center ErlangenEMN, Friedrich-Alexander University Erlangen-Nuremberg, Erlangen, Germany

2 Department of Gynecology and Obstetrics, Ulm University Hospital, Ulm, Germany

3 Department of Obstetrics and Gynecology, University of Tübingen, Tübingen, Germany

4 Oncologianova GmbH, Recklinghausen, Germany

5 Marienhospital Bottrop, Bottrop, Germany

6 Department of Bone Oncology, Nordwest Hospital, Frankfurt, Germany

7 Oncology Practice at Bethanien Hospital Frankfurt, Frankfurt, Germany

8 Department of Obstetrics and Gynecology, Klinikum rechts der Isar, Technical University of Munich, Munich, Germany

9 Charité University Hospital, Berlin, Campus Benjamin Franklin, Department of Hematology, Oncology and Tumour Immunology, Berlin, Germany

10 Department of Obstetrics and Gynecology, University of Heidelberg, Heidelberg, Germany

11 Department of Gynecology, Hamburg-Eppendorf University Medical Center, Hamburg, Germany

12 ClinSol GmbH \& Co. KG, Würzburg, Germany

13 Department of Gynecology and Obstetrics, University Hospital Düsseldorf, Düsseldorf, Germany

14 National Center for Tumor Diseases, Division Gynecologic Oncology, University Hospital Heidelberg, Heidelberg, Germany

Key words

breast cancer, treatment, local recurrence, metastases, studies

Schlüsselwörter

Mammakarzinom, Behandlung, Lokalrezidiv, Metastasen, Studien $\begin{array}{ll}\text { received } & 4.11 .2017 \\ \text { revised } & 13.11 .2017 \\ \text { accepted } & 13.11 .2017\end{array}$

Bibliography

DOI https://doi.org/10.1055/s-0043-122885

Geburtsh Frauenheilk 2017; 77: 1281-1290 @ Georg Thieme

Verlag KG Stuttgart · New York | ISSN 0016-5751

Correspondence

Peter A. Fasching, MD

Erlangen University Hospital, Department of Gynecology

and Obstetrics, Comprehensive Cancer Center Erlangen EMN, Friedrich Alexander University of Erlangen-Nuremberg Universitätsstraße 21-23, 91054 Erlangen, Germany

peter.fasching@uk-erlangen.de

$\Theta$ Deutsche Version unter: https://doi.org/10.1055/s-0043-122885

\section{ABSTRACT}

In recent years, numerous new therapy options for patients with breast cancer have been developed in clinical studies, with some options already approved for routine treatment. As the speed at which innovations are introduced increases, the importance of conferences also increases, as conferences are where the data underpinning new therapies are usually presented for the first time. This review looks at publications of the ASCO (American Society of Clinical Oncology) and ESMO (European Society of Medical Oncology) conferences in 2017, summarizes them and evaluates them in the context of existing data. The focus is on new insights for neoadjuvant therapy and new treatment options in the metastatic setting, such as the use of CDK4/6 inhibitors or PARP inhibitors. The first results of treatments with checkpoint inhibitors are presented. With the patent expiry of trastuzumab, a number of study results for trastuzumab biosimilars have also been published. The digitization of patient care provides the first results on quality of life and prognosis of patients with advanced 
cancer. Digital communications between patients and physicians are being evaluated in several studies in Germany. As the discussion about patient-relevant endpoints for patients in the metastatic setting continues, overall survival rates from studies of big endocrine-based therapies are urgently needed. Preliminary analyses of small study cohorts offer initial insights. In the context of improving patient care, in the coming years, questions will center on which patients particularly benefit from certain therapies and which patients need particular protection from specific side effects. Questions about these predictors are raised in many scientific projects as attention increasingly focuses on this topic.

\section{ZUSAMMENFASSUNG}

In den letzten Jahren sind zahlreiche neue Therapieoptionen für die Patientin mit einem Mammakarzinom in klinischen Studien entwickelt worden und teilweise zur Zulassung gekommen. Bei zunehmender Geschwindigkeit neuer Innovationen nimmt die Bedeutung von Kongressen zu, auf denen meist zum ersten Mal Daten zu den entsprechenden Therapien präsentiert werden. Diese Übersichtsarbeit greift Veröffentlichungen der Kongresse ASCO (American Society of Clincial Oncology) und ESMO (European Society of Medical Oncology) aus dem Jahr 2017 auf und fasst sie im Kontext be- stehender Daten zusammen. Der Fokus liegt auf Erkenntnissen aus der neoadjuvanten Situation und Neuigkeiten aus der metastasierten Situation, wie der Einsatz von CDK4/6-Inhibitoren oder PARP-Inhibitoren. Des Weiteren werden erste Ergebnisse zur Behandlung mit Checkpoint-Inhibitoren dargestellt. Bei baldigem Patentablauf von Trastuzumab sind zudem einige Studienergebnisse zu Trastuzumab-Biosimilars veröffentlicht worden. Auch die Digitalisierung der Patientenversorgung liefert erste Ergebnisse in Bezug auf Lebensqualität und Prognose von Patientinnen mit fortgeschrittener Krebserkrankung. Die digitale Kommunikation zwischen Patientin und Ärztin bzw. Arzt wird auch in Deutschland in einigen Studien untersucht. Bei anhaltender Diskussion über patientenrelevante Endpunkte für Patientinnen in der metastasierten Situation werden die Gesamtüberlebensdaten für die großen endokrin basierten Studien dringend erwartet. Erste Einblicke liefern vorläufige Analysen an kleinen Studienkollektiven. Im Rahmen der Patientenversorgung werden in den nächsten Jahren die Fragen aufkommen, welche Patientinnen besonders von bestimmten Therapien profitieren und welche vor besonders relevanten Nebenwirkungen geschützt werden können. Die Frage nach diesen Prädiktoren begleitet viele wissenschaftliche Projekte und bekommt eine besondere Aufmerksamkeit.

\section{Introduction}

A number of targeted drugs have been developed to clinical approval in recent years. Other drugs are in advanced stages of development. They include inhibitors of the PI3K/Akt-kinase signaling pathway, such as mTOR (mammalian target of rapamycin) inhibitors and PI3K (phosphatidylinositol 3-kinase) inhibitors, inhibitors of the PARP (poly [ADP-ribose] polymerase) enzyme, immunomodulators such as antibodies against PD-1 (programmed cell death protein-1) and PD-L1 (programmed cell death protein-1/ ligand-1) and, not least, CDK (cyclin-dependent kinase) 4/6 inhibitors, which inhibit the cell cycle in hormone receptor-positive cancer. These drugs are a consequence of the growing understanding of both cellular functions and the genetics of cancers and of patients' hereditary genetics. This article summarizes new data publicized in 2017 at the two big international conferences ASCO and ESMO, focuses particularly on the most recent medical therapies for patients with breast cancer.

\section{Neoadjuvant Therapy}

Treating patients with primary breast cancer prior to surgery (i.e., neoadjuvant therapy) continues to be hugely important in routine treatment, the development of new drug therapies and the modification of existing therapy concepts. While it has been possible to establish a link between pathological complete remission $(p C R)$ and excellent prognosis for some molecular subtypes (triple-negative, HER2-positive), this association has not been clearly proven for hormone receptor-positive, HER2-negative breast cancer $[1,2]$. There are some indications that the link between $p C R$ and prognosis in certain molecular groups, e.g. patients with BRCA (breast cancer gene) mutations, is not as pronounced as when these molecular changes are not present [3]. It is therefore particularly interesting to see how pronounced the connection between PCR and prognosis is in the most recent studies.

In this context this year saw the presentation of updated survival data from the NeoALTTO study [4]. With a median follow-up of 6.7 years, the study confirmed that, irrespective of the therapy, pCR is an excellent predictor of recurrence-free survival and overall survival for HER2-positive breast cancer. The study which compared neoadjuvant therapy with paclitaxel plus either lapatinib, lapatinib + trastuzumab or trastuzumab, was not able to show any differences in survival variables [4], despite the differences in pCR [5]. The final survival data from the similar GeparQuinto trial [6] have not yet been published.

The GeparSixto trial [7], which evaluated the benefits of adding carboplatin to anthracycline-based and taxane-based chemotherapy to treat patients with triple-negative and HER2-positive primary cancer, was able to show the effect of therapy on pCR and on the subsequent prognosis. The addition of carboplatin significantly improved the prognosis of patients with triple-negative breast cancer ( $\mathrm{HR}=0.56$ [95\% Cl: $0.34-0.93]$; $\mathrm{p}=0.024)$ but had no significant impact on HER2-positive breast cancer [8]. Platinum-based treatment should therefore increasingly become standard treatment in the neoadjuvant setting for patients with triplenegative breast cancer.

In the GeparOcto trial, one arm of the trial compared platinumbased chemotherapy (weekly paclitaxel, liposomal doxorubicin and carboplatin) with dose-dense chemotherapy with epirubicin, paclitaxel and cyclophosphamide (ETC regimen) in triple-nega- 
tive, HER2-positive and high-risk hormone receptor-positive/ HER2-negative patients. HER2-positive patients additionally received dual therapy with trastuzumab and pertuzumab. No differences were found in PCR rates [9]. The survival data have not yet been published.

A neoadjuvant study which contained the anti-PD-1 antibody pembrolizumab is described below in the chapter "Immuno-Oncology".

The development of therapies often requires testing for molecular markers. While testing for hormone receptor status and HER2 status often only takes a few days, testing for other markers such as BRCA1/2 mutations or for characteristics often summarized by the term "homologous repair deficiency (HRD)" can take several weeks. The question then arises whether the wait to obtain a precise molecular characterization could worsen the prognosis of women with breast cancer. An analysis of more than 8000 women with breast cancer did not find that the time between diagnosis and the start of neoadjuvant chemotherapy or the time between the end of chemotherapy and surgery had an impact on recurrence-free survival or overall survival as long as the cut-off was 4 weeks [10]. This indicates that there is no obstacle to carrying out more comprehensive molecular testing, even if it takes longer than determining hormone receptor and HER2 status. The GeparOLA trial, which is currently still recruiting and randomizes patients with BRCA1/2 germline mutations or tumors and patients with identified HRD for chemotherapy with or without the PARP inhibitor olaparib, has adopted this approach [11].

\section{Adjuvant Therapy}

Researchers are keenly awaiting data from the APHINITY trial, one of the biggest currently running adjuvant therapy trials with antibodies $[12,13]$. This German-led trial is investigating the effect of pertuzumab in addition to treatment with trastuzumab, as previous studies appeared to indicate that in the metastatic and neoadjuvant setting pertuzumab combined with trastuzumab and chemotherapy was more effective than trastuzumab and chemotherapy alone. In the metastatic setting, the addition of pertuzumab significantly improved both recurrence-free survival (18.5 vs. 12.4 months) and overall survival (56.5 vs. 40.8 months) $[14,15]$. In the neoadjuvant setting, the NeoSphere trial showed a rise in the pCR rate (ypT0/ypN0) from 23 to $42 \%$ [16]. Based on these data, the APHINITY trial was initiated, with more than 4800 participants. Randomization was done $1: 1$ with patients randomized to receive standard chemotherapy with trastuzumab or dual antibody therapy with the addition of pertuzumab. Primary endpoint of the study was invasive recurrence-free survival. Although the overall prognosis for both treatment arms was excellent, the trial still showed a small but significant benefit when pertuzumab was added. After a median follow-up of 45 months, 210 events (invasive 4-year DFS: $90.6 \%$ ) had occurred in the trastuzumab arm while 171 events (invasive 4-year DFS: 92.3\%) had occurred in the pertuzumab-trastuzumab arm. This resulted in a hazard ratio of 0.81 (95\% Cl: 0.66-1.00; $p=0.045)$ [12,13]. The clinical importance of these data and the question whether pertuzumab should be administered to the total study population or to special sub- groups (e.g. with node-positive, hormone receptor-negative cancers) will need to be discussed in the context of its approval for use.

Other anti-HER2 substances are also being tested in the adjuvant setting. Results from the ExteNET trial with a follow-up of 2 years have already been published [17] and showed an improvement in invasive recurrence-free survival when patients with HER2-positive breast cancer who had had one year of treatment with trastuzumab were subsequently treated with the small molecule neratinib for one year. The 5-year survival rates were published at this year's ESMO 2017 conference [18]. With a hazard ratio of 0.78 ( $95 \% \mathrm{Cl}: 0.57-0.92 ; \mathrm{p}=0.008)$ the results were similar to those in the initial publication $(\mathrm{HR}=0.67)$. Researchers are currently awaiting the approval of further trials. It will be necessary to develop strategies to identify those patient characteristics which indicate that patients with these characteristics should receive the expanded targeted therapy. A subgroup analysis showed a particular benefit for hormone receptor-positive patients.

\section{New Treatment Options in the Metastatic Setting}

\section{CDK4/6 inhibitors}

In the last two years, a wealth of data has been published on the use of CDK4/6 inhibitors to treat patients with hormone receptorpositive, HER2-negative metastatic breast cancer [19-22]. Up to now, data were only available for the CDK4/6 inhibitors palbociclib and ribociclib, both of which have been approved for use in Germany. However, this year, data on the CDK4/6 inhibitor abemaciclib was presented at the 2017 ASCO and ESMO conferences [23-26]. While the MONARCH 2 trial included patients, who had recurrence during neoadjuvant or adjuvant endocrine therapy or within 12 months of completing therapy, the MONARCH 3 trial focused on patients who had not yet received systemic therapy for advanced or metastatic disease.

In the MONARCH 2 trial patients were randomized into treatment with fulvestrant or treatment with fulvestrant + abemaciclib. Progression-free survival (PFS) with monotherapy was 9.3 months, but PFS survival was increased to 16.4 months with the combination therapy $(\mathrm{HR}=0.553 ; 95 \% \mathrm{Cl}: 0.449-0.681$; $\mathrm{p}<0.001)$. Subgroup analysis found no particular group which benefited especially well or less well from therapy with abemaciclib. The most common side effect of treatment with abemaciclib was neutropenia (grade 3 and 4), which occurred in $23.6 \%$ of patients compared with $1.3 \%$ in the group of patients who received fulvestrant monotherapy. Diarrhea (grade 3 and 4) was the second most common side effect in the group which received fulvestrant + abemaciclib, affecting $13.4 \%$ of patients compared to just $0.4 \%$ of patients in the group which received fulvestrant monotherapy [24].

The MONARCH 3 trial randomized patients to receive either treatment with a non-steroidal aromatase inhibitor (nsAl; anastrozole or letrozole) or treatment with nsAl + abemaciclib. The addition of abemaciclib significantly extended PFS (HR $=0.54 ; 95 \% \mathrm{Cl}$ : 0.41-0.72; $p=0.00021)$. While the median PFS with fulvestrant 
OS: Phase 2 (ITT)

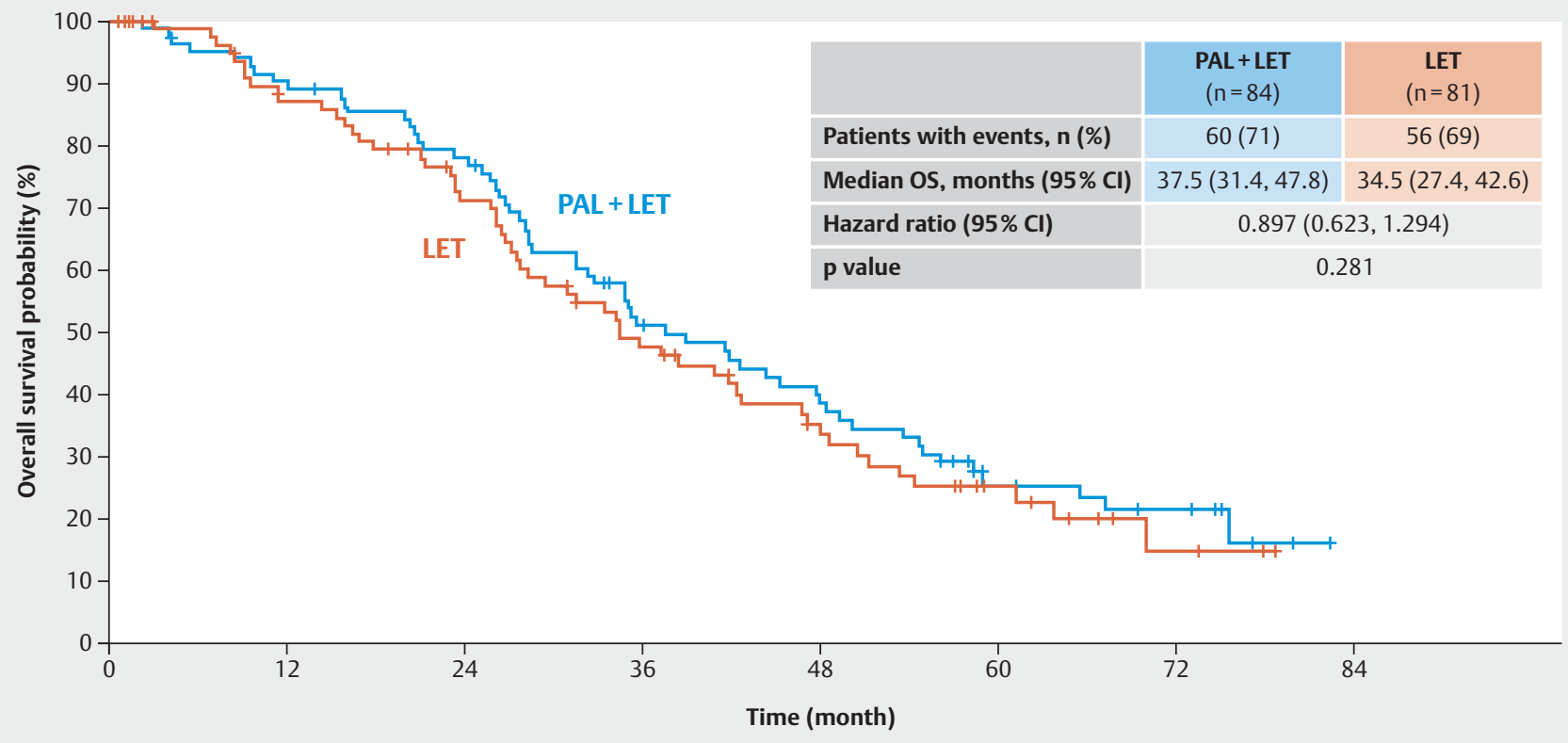

Number of patients at risk

$\begin{array}{lllllll}84 & 73 & 63 & 38 & 28 & 13 & 8 \\ 81 & 67 & 52 & 33 & 21 & 10 & 3\end{array}$

- Fig. 1 Overall survival in the PALOMA 1 trial, modified according to [27].

was 14.7 months, the median PFS has not yet been reached for treatment with nsAl + Abemaciclib [24]. A subgroup analysis in this study also did not find any group of patients who benefited especially from treatment. The side-effect profile was similar to that reported in the MONARCH 2 trial (9.5\% grade 3 and 4 diarrhea; $21.1 \%$ grade 3 and 4 neutropenia) [24].

Even though many studies have demonstrated the significant benefits of CDK4/6 inhibitors with regard to PFS [20-24], data on overall survival (OS) are currently still lacking. Overall survival results have been reported for the PALOMA 1 trial and the MONALEESA 2 trial but have not yet been published in full ( $\triangleright$ Figs. 1 and 2). At the ASCO 2017 conference, the PALOMA 1 trial, which only randomized 165 patients and received provision approval in the USA based on this data, reported a median OS of 34.5 months for letrozole alone and median OS of 37.5 months for letrozole combined with palbociclib [27]. The hazard ratio was 0.897 (95\% Cl: 0.623-1.294; $p=0.281$ ) [27]. Analysis in the MONALEESA 2 trial showed that the median OS time had not yet been reached. With an HR of 0.746 (95\% Cl: $0.517-1.076$; $\mathrm{p}=0.059)$ the trial currently still just misses statistical significance [28]. Data which would show an overall survival benefit are therefore still lacking. It should, however, be noted that all of these studies are underpowered regarding a median OS of around 50 months which is currently achieved in several current studies of women with hormone receptor-positive metastatic breast cancer [29, 30].

Therapies with CDK4/6 inhibitors have prompted a renewed discussion about patient-relevant endpoints. The most intensely discussed issue in this context is whether overall survival is the only relevant objective of a study or would a significant benefit with regard to progression-free survival be sufficient? In their latest presentation of the PALOMA 1 trial, Finn et al. also presented an analysis of the time to first chemotherapy following randomization. They were able to show that the time to chemotherapy increased significantly from a median of 17.7 months with letrozole to 26.7 months with letrozole + palbociclib $(\mathrm{HR}=0.662[95 \% \mathrm{Cl}$ : 0.445-0.989, > Fig. 3]). Based on the existing data and the clinically relevant significant extension of PFS, the use of CDK4/6 inhibitors has become standard to treat hormone receptor-positive metastatic breast cancer, which is reflected in the guidelines and recommendations.

\section{Homologous repair deficiency: patient-oriented therapies and other hereditary panel genes}

$B R C A 1$ and BRCA2 are DNA repair genes. They are involved in the repair of DNA double-strand breaks (homologous repair). PARP (poly [adenosine diphosphate ribose]) enzymes are essential for the repair of DNA single-strand breaks [31]. In the preclinical stage, cancer cells with a BRCA1/2 mutation are sensitive to PARP inhibitors. The hypothesis is that in women with BRCA1/2 mutation, the additional inhibition of another DNA repair mechanism leads to synthetic lethality. PARP inhibitors which are either in clinical development or have already been approved include talazoparib (BMN673 or MDV3800), olaparib (AZD2281 or KU0059436), niraparib (MK4827), veliparib (ABT888) and rucaparib 


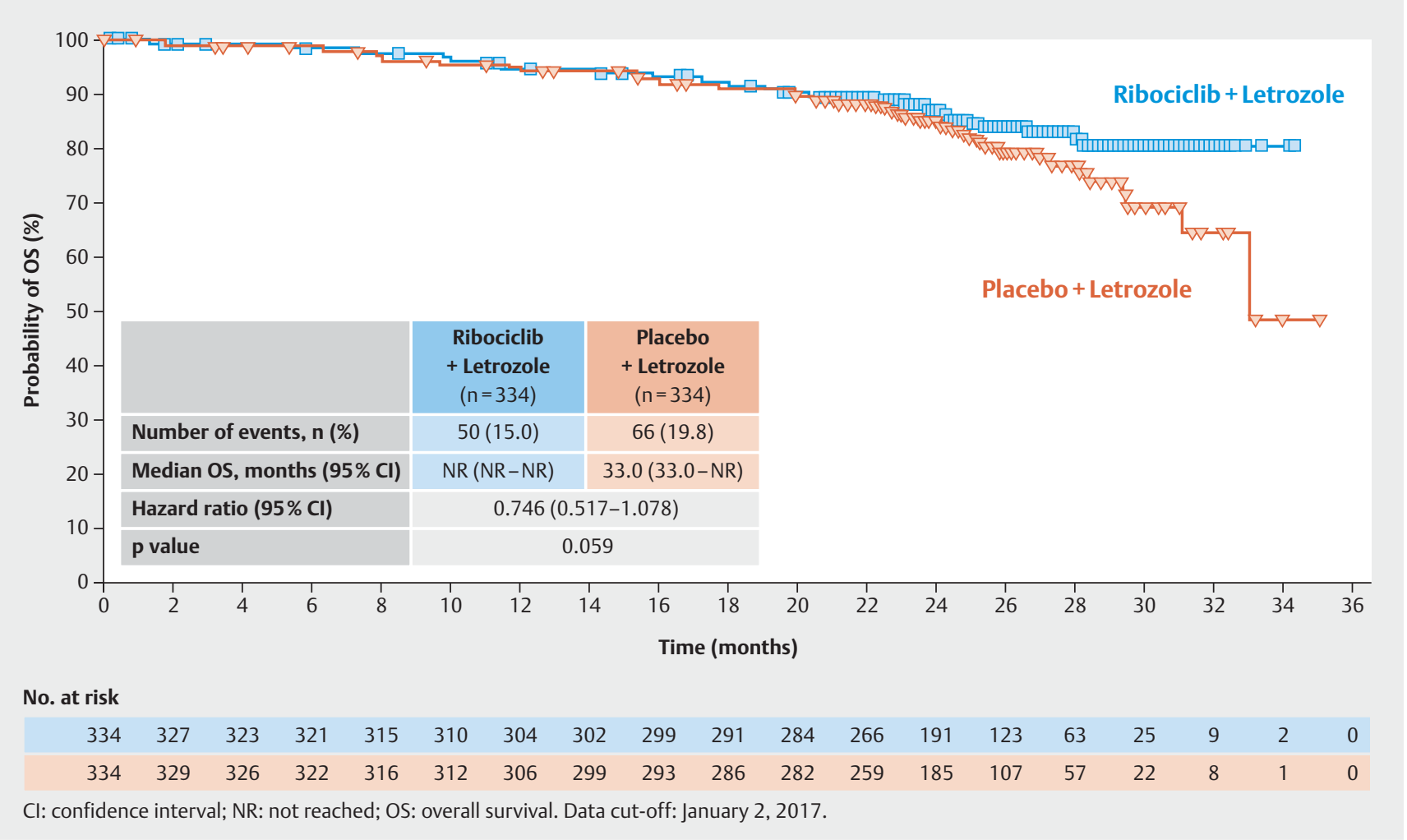

- Fig. 2 Overall survival in the MONALEESA 2 trial, modified according to [28].

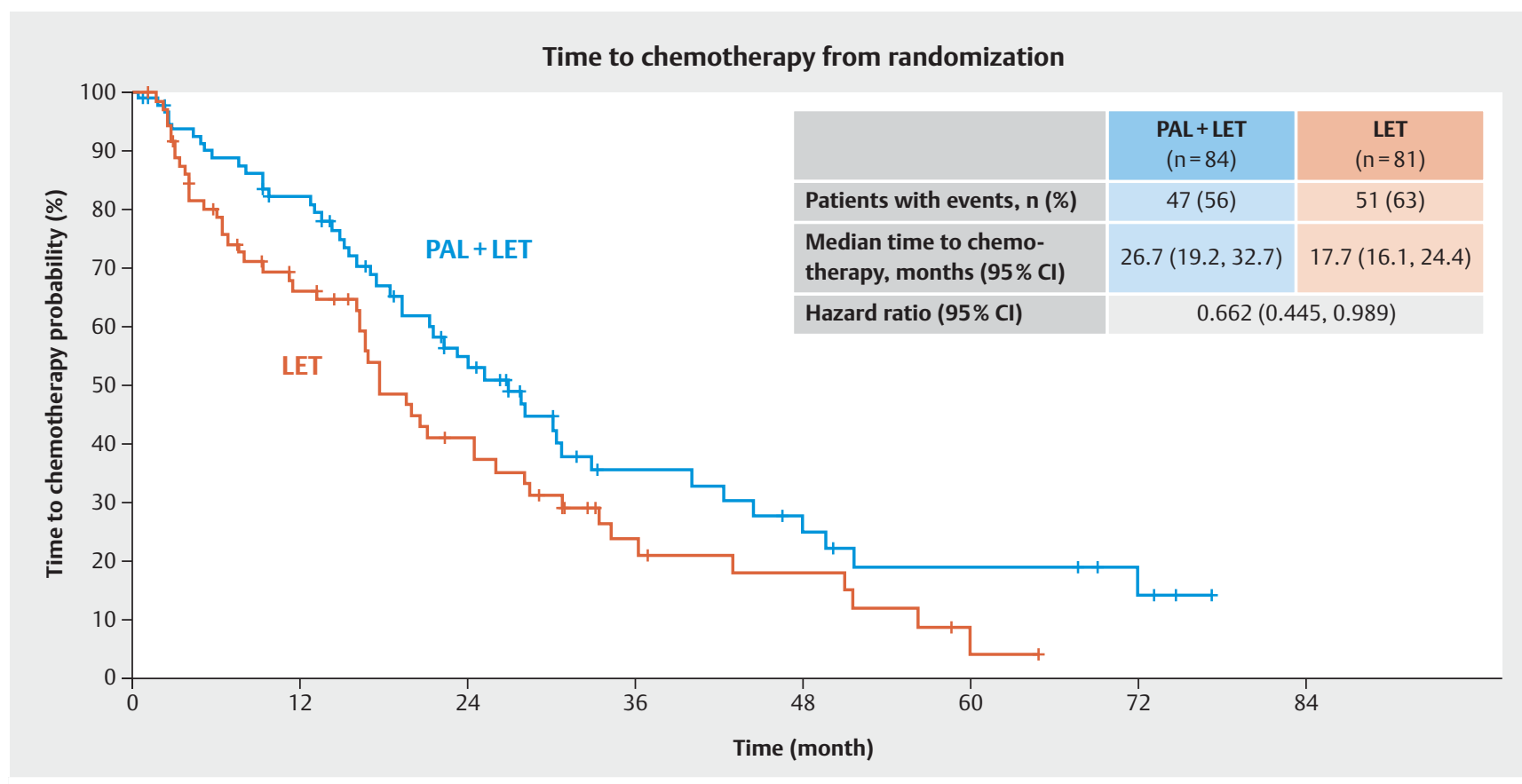

- Fig. 3 Time to chemotherapy in the PALOMA 1 trial, modified according to [27]. 
(AG-014699 or PF-01367338). In the USA and Europe olaparib has already been approved to treat certain patients with ovarian cancer (with BRCA1/2 mutations and platinum-sensitive recurrence). The PARP inhibitor niraparib has been approved for use in the USA to treat women with platinum-sensitive ovarian cancer recurrence irrespective of whether patients have BRCA mutation or not. The European Medicines Agency has also already voted in favor of extending the approval.

Outcomes from the OlympiaD trial, an olaparib approval study for metastatic breast cancer [32,33], were presented at the ASCO 2017 conference. The study randomized 302 patients with germline $B R C A 1$ or $B R C A 2$ mutations. For inclusion in the study, patients should not have had more than 2 previous chemotherapies. Hormone receptor-positive patients had to have had at least one line of endocrine therapy [32]. The trial compared monotherapy with olaparib (2:1 randomization) with a chemotherapy chosen by the treating physician (either capecitabine, eribulin or vinorelbin). In this patient population, which per se had a poor prognosis, the median progression-free interval of 4.2 months with chemotherapy improved to 7.0 months with olaparib monotherapy $(\mathrm{HR}=0.58$; $95 \% \mathrm{Cl}: 0.43-0.80 ; \mathrm{p}=0.0009)[32,33]$. No statistically significant improvement in overall survival was found between the two randomization arms in the study $(\mathrm{HR}=0.90 ; 95 \% \mathrm{Cl}$ : $0.63-1.29 ; p=0.57$ ) [32]. Subgroup analysis found no clinically relevant differences between groups [32-34]. Anemia and neutropenia were the most commonly reported side effects of treatment with olaparib, with respective rates of $16.4 \%$ (grade 3 and 4 ) and $9.3 \%$ (grade 3 und 4); however rates of anemia and neutropenia were even higher with chemotherapy (26.4 and 49.5\%) [32]. Given this clinically relevant benefit of monotherapy with the PARP inhibitor compared to chemotherapy, approval is eagerly anticipated as it appears to offer a further promising treatment option for this special cohort of patients.

The ABRAZO trial, a study which also had a number of participating German centers, evaluated the benefits of PARP inhibitor talazoparib to treat patients with germline BRCA1/2 mutations, with the results presented at the 2017 ASCO conference [35]. ABRAZO is a single-arm study with 2 cohorts. One cohort recruited patients who showed a response to platinum-based chemotherapy in the metastatic setting and the other cohort consisted of patients who had received 3 or more chemotherapies in a metastatic setting but had not received platinum-based treatment. The median PFS of cohort 1 was 4 months (95\% Cl: $2.8-$ 5.4 months), while the median PFS of cohort 2 was 5.6 months (95\% Cl: 5.5-7.8 months) [35]. Once again, the most commonly reported side effects of this PARP inhibitor were anemia (grade 3 and 4 in 16\% of patients) and neutropenia (grade 3 and 4 in 6\%). In addition, grade $3 / 4$ thrombocytopenia was reported for $10 \%$ of patients [35].

The importance of PARP inhibitors compared to platinumbased therapies was evaluated in the Brightness study, with new data presented at the ESMO 2017 conference [36]. This neoadjuvant study compared 634 patients with triple-negative breast cancer (BRCA mutation was not an inclusion criteria) in three treatment arms: paclitaxel followed by doxorubicin + cyclophosphamide vs. paclitaxel and carboplatin followed by doxorubicin + cyclophosphamide vs. paclitaxel + carboplatin + veliparib followed by doxorubicin + cyclophosphamide. The pCR rates for the three regimens were 31.0 vs. 57.5 vs. $53.2 \%$ [36], although the difference between the two therapies which included carboplatin was not statistically significant and the addition of veliparib did not result in any improvement in the $\mathrm{PCR}$ rate and is therefore currently not recommended ( $p=0.357$ ).

Patient selection based on molecular changes and the type of previous platinum-based therapy appears to play an important role in treatments with PARP inhibitors. Achieving a better understanding of which patients are best suited to receive particular therapies will be extremely important to successfully establish these therapies in clinical practice. It is still not clear, for example, what role other genes currently being evaluated in panel gene tests may play. It is known, for example, that ATM, CHEK2, PALB2, $B A R D 1$ and RAD51D play a similar role to that of BRCA1 and BRCA2 in the repair of DNA double-strand breaks. Mutations occur in breast cancer with a frequency that indicates that it would be useful to take these mutations into consideration when treating patients with breast cancer $[37,38]$. The importance of other lowpenetrance cancer predisposition genes has not yet entered into therapy planning. A recent publication which carried out a genome-wide association study of more than 122000 cases with breast cancer and more than 105000 controls was able to show that the low-penetrance variants identified to date make up around $18 \%$ of the familial risk for breast cancer [39]. Some of these variants are particularly common in patients with triplenegative breast cancer [ $40-43]$.

\section{Immuno-Oncology}

The KEYNOTE-173 trial, presented at the ASCO 2017 conference, has been evaluating the checkpoint inhibitor pembrolizumab in two cohorts in a neoadjuvant setting. With 10 patients each per treatment cohort (no randomization), this study provides initial data on PCR rates following chemotherapy combined with the anti-PD-1 antibody pembrolizumab [44]. Treatment with nabpaclitaxel followed by doxorubicin and cyclophosphamide in combination with pembrolizumab resulted in complete remission of all tumor cells in $50 \%$ of patients. The PCR rate for a chemotherapy regimen which additionally included carboplatin (in combination with nab-paclitaxel) was $80 \%$ [44].

The KEYNOTE-086 trial studied patients with triple-negative metastatic breast cancer who had been previously treated in the metastatic setting and had tested positive for PD-L1 in the tumor. The analysis of the results for 52 patients was available at the time of presenting the poster. With a median follow-up time of 7 months, 15 patients (29\%) were still receiving monotherapy with pembrolizumab [45].

Even though these two studies found no persisting side effects, it is importance to establish treatment of new types of side effects in routine clinical practice. Particularly neuroendocrine side effects require better training of medical staff to make innovative therapies safer in clinical practice. 
- Table 1 Antibodies which have been tested as biosimilars for trastuzumab.

\begin{tabular}{|l|l|l|l|l|l|l|l|}
\hline Name & Manufacturer & N & $\begin{array}{l}\text { PCR with } \\
\text { trastuzumab }\end{array}$ & $\begin{array}{l}\text { PCR with } \\
\text { biosimilar }\end{array}$ & RR or OR & Interpretation & Reference \\
\hline ABP980 & Amgen & 827 & $41.8 \%$ & $47.8 \%$ & $1.14(90 \% \mathrm{Cl}: 0.993-1.312)$ & equivalent & {$[52]$} \\
\hline CT-P6 & Celltrion & 549 & $50.4 \%$ & $46.8 \%$ & $0.9282(95 \% \mathrm{Cl}$ : 0.775-1.111) & equivalent & {$[46,51]$} \\
\hline SB3 & Samsung/Merck-MSD & 800 & $42.0 \%$ & $51.7 \%$ & $1.259(90 \% \mathrm{Cl}: 1.112-1.426)$ & equivalent & {$[47,50]$} \\
\hline PF-05280014 & Pfizer & 226 & $50.0 \%$ & $47 \%$ & not reported & equivalent & {$[49]$} \\
\hline
\end{tabular}

\section{Biosimilars}

With the expiry of the patent for trastuzumab, a number of biosimilar studies of anti-HER2 antibodies were presented at this year's conferences [46-52]. Four different antibodies are expected to be introduced in Germany in 2018. A neoadjuvant setting is the most common scenario used to test biosimilars. The presented studies are summarized in $>$ Table 1 . In all studies biosimilars were evaluated for their equivalence to trastuzumab in terms of the PCR rates achieved. None of the studies reported any difference in their profile of side effects or any other more common side effects compared to trastuzumab [46 - 52].

One study on PF-05280014 reported on its use in 707 patients as a first-line treatment for metastatic breast cancer. The reported progression-free 1 -year survival was $52 \%$ for trastuzumab and $56 \%$ for PF-05280014. The profile of side effects for PF05280014 was similar to that of trastuzumab [48].

\section{Patient Management}

Physicians have long focused on compliance and the management of side effects in endocrine therapy in both adjuvant and metastatic settings [53-55]. As new endocrine combination therapies are being developed, attention has once again begun to focus on these issues. As we move into the digital age, research has also begun to look at new ways of involving patients in their medical care. A prospective randomized study which recruited patients receiving chemotherapy for advanced breast cancer or genitourinary, gynecological or lung cancer $(n=766)$ showed that the use of internet-based monitoring improved overall survival [56]. The patients who were randomized into the intervention arm were asked to self-report on twelve common symptoms before and after hospital visits using a web-based monitoring system. In the other randomization arm, side effects were only recorded in accordance with standard clinical care. The authors reported a significantly better overall survival (HR: 0.832; $95 \% \mathrm{Cl}$ : 0.696-0.995) following self-reporting using a web-based system. The study also found fewer emergency room visits and hospitalizations in the group using the self-reporting system [56].

It is not yet clear which mechanisms resulted in the higher survival rates reported in their study. However, it is known that side effects can have an impact on therapy compliance. This, in turn, could lead to a poorer prognosis. It has been reported in this context that untreated side effects result in poorer compliance, particularly in endocrine therapies $[54,57,58]$.
A few study concepts in Germany have been proposed to evaluate the potential benefits of electronic medical technologies (eHealth) which use modern internet-based systems to support patients. The PreCycle study will be randomizing patients receiving palbociclib into two groups: one group will use a web-based interaction tool (CANKADO) and one group will receive standard care [59]. The PRAEGNANT study and the Seraphina study will also be investigating the impact of paper-based and web-based patient-reported outcomes (PRO) for patients with metastatic breast cancer [60-64].

\section{Conclusion}

With the introduction of CDK4/6 inhibitors, PARP inhibitors and immunotherapies, new classes of substances have been approved for treatment or are in advanced stages of clinical development. As for all substances which are just starting to be introduced into routine clinical practice, the question arises to what extent it will be possible to identify patients who would particularly benefit from these new therapies and which patients might need to be particularly protected from side effects. In the next few years, it will hopefully be possible to establish clinical or molecular predictors for some of these substances which will help physicians weigh up the options.

\section{Acknowledgements}

This article was partly funded by Pfizer. Pfizer had no share in compiling the manuscript. Sole responsibility for the contents of this manuscript lies with the authors.

\section{Conflict of Interest}

A.D. H. received honoraria from Teva, GenomicHealth, Celgene, AstraZeneca, Novartis, Pfizer and Roche. N. N. received consultancy honoraria from Janssen-Cilag and travel support from Novartis. F. O. received speaker and consultancy honoraria from Amgen, Celgene, AstraZeneca, Novartis, Roche, and MSD. H.-C. K. received honoraria from Carl Zeiss meditec, TEVA, Theraclion, Novartis, Amgen, AstraZeneca, Pfizer, Janssen-Cilag, GSK, LIV Pharma and Genomic Health. P. H. received honoraria, unrestricted educational grants and research funding from Amgen, AstraZeneca, Eli Lilly, MSD, Novartis, Pfizer and Roche. P. A. F. received honoraria from Roche, Pfizer, Novartis and Celgene. His institution conducts research for Novartis. H. T. received honoraria from Novartis, Roche, Celgene, TEVA, Pfizer and travel support from Roche, Celgene and Pfizer. J. E. received honoraria from Roche, Celgene, Novartis, Pfizer, Pierre Fabre, TEVA and travel support from Celgene, Pfizer, TEVA and 
Pierre Fabre. M. P. L. received honoraria from Pfizer, Roche, MSD, Hexal, Novartis, Lilly, AstraZeneca, TEVA, Celgene, Eisai, medac and Thieme for advisory boards, lectures and travel support. M. W. received speaker honoraria from AstraZeneca, Celgene and Novartis. V. M. received speaker honoraria from Amgen, AstraZeneca, Celgene, Daiichi-Sankyo, Eisai, Pfizer, Pierre-Fabre, Novartis, Roche, Teva, Janssen-Cilag and consultancy honoraria from Genomic Health, Roche, Pierre Fabre, Amgen, Daiichi-Sankyo and Eisai. E. B. received honoraria from Novartis for consulting and clinical research management activities. C. H. received honoraria from Amgen, Celgene, Oncovis, Roche and Pfizer. A. S. received honoraria from Roche, Celgene, AstraZeneca, Novartis, Pfizer, Zuckschwerdt Verlag GmbH, Georg Thieme Verlag, Aurikamed $\mathrm{GmbH}, \mathrm{MCl}$ Deutschland $\mathrm{GmbH}$, bsh medical communications $\mathrm{GmbH}$ and promedicis $\mathrm{GmbH}$. W. A. received honoraria from Amgen, AbbVie, Bendalis, BMS, Celgene, IOMEDICO, Gilead, GSK, Lilly, MSD, Novartis, Pfizer, Roche, Hexal, TEVA. W.J. received honoraria and research grants from Novartis. D. L. received honoraria from Amgen, Celgene, Daiichi Sankyo, Eli Lilly, Loreal, MSD, Novartis, Pfizer, Roche. All other authors have declared no conflicts of interest.

\section{References}

[1] von Minckwitz G, Untch M, Blohmer JU et al. Definition and impact of pathologic complete response on prognosis after neoadjuvant chemotherapy in various intrinsic breast cancer subtypes. J Clin Oncol 2012; 30: 1796-1804

[2] Cortazar P, Zhang L, Untch M et al. Pathological complete response and long-term clinical benefit in breast cancer: the CTNeoBC pooled analysis. Lancet 2014; 384: 164-172

[3] Paluch-Shimon S, Friedman E, Berger R et al. Neo-adjuvant doxorubicin and cyclophosphamide followed by paclitaxel in triple-negative breast cancer among BRCA1 mutation carriers and non-carriers. Breast Cancer Res Treat 2016; 157: 157-165

[4] Huober JB, McCormick Holmes E, Baselga J et al. Survival outcomes of the NeoALTTO study: updated results of a randomized multicenter phase III neoadjuvant trial. J Clin Oncol 2017; 35 (Suppl.): Abstr. 512

[5] Baselga J, Bradbury I, Eidtmann $\mathrm{H}$ et al. Lapatinib with trastuzumab for HER2-positive early breast cancer (NeoALTTO): a randomised, open-label, multicentre, phase 3 trial. Lancet 2012; 379: 633-640

[6] Untch M, Loibl S, Bischoff J et al. Lapatinib versus trastuzumab in combination with neoadjuvant anthracycline-taxane-based chemotherapy (GeparQuinto, GBG 44): a randomised phase 3 trial. Lancet Oncol 2012; 13: $135-144$

[7] von Minckwitz G, Schneeweiss A, Loibl S et al. Neoadjuvant carboplatin in patients with triple-negative and HER2-positive early breast cancer (GeparSixto; GBG 66): a randomised phase 2 trial. Lancet Oncol 2014; 15: 747-756

[8] Untch M, Schneeweiss A, Salat C et al. Long-term survival analysis of the randomized phase II trial investigating the addition of carboplatin to neoadjuvant therapy for triple-negative (TNBC) and HER2-positive early breast cancer (GeparSixto). Ann Oncol 2017; 28 (Suppl. 5): v43-v67, Abstr. 163PD

[9] Schneeweiss A, Moebus V, Tesch $\mathrm{H}$ et al. A randomised phase III trial comparing two dose-dense, dose-intensified approaches (EPC and PM (Cb)) for neoadjuvant treatment of patients with high-risk early breast cancer (GeparOcto). J Clin Oncol 2017; 35 (Suppl.): Abstr. 518

[10] Loibl S, Werutsky G, Nekljudova $V$ et al. Impact in delay of start of chemotherapy and surgery on PCR and survival in breast cancer: a pooled analysis of individual patient data from six prospectively randomized neoadjuvant trials. J Clin Oncol 2017; 35 (Suppl.): Abstr. 571
[11] Fasching PA, Blohmer JU, Burchardi $\mathrm{N}$ et al. A randomized phase II trial to assess the efficacy of paclitaxel and olaparib in comparison to paclitaxel/ carboplatin followed by epirubicin/cyclophosphamide as neoadjuvant chemotherapy in patients with HER2-negative early breast cancer and homologous recombination deficiency (HRD): GeparOLA. J Clin Oncol 2016; 34 (Suppl.): Abstr. TPS1096

[12] von Minckwitz G, Procter M], De Azambuja E et al. APHINITY trial (BIG 4 11): a randomized comparison of chemotherapy $(C)$ plus trastuzumab ( $\mathrm{T}$ ) plus placebo (Pla) versus chemotherapy plus trastuzumab $(\mathrm{T})$ plus pertuzumab $(\mathrm{P})$ as adjuvant therapy in patients (pts) with HER2-positive early breast cancer (EBC). J Clin Oncol 2017; 35 (Suppl.): Abstr. LBA500

[13] von Minckwitz G, Procter M, de Azambuja E et al. Adjuvant pertuzumab and trastuzumab in early HER2-positive breast cancer. N Engl J Med 2017; 377: 122-131

[14] Baselga J, Cortes J, Kim SB et al. Pertuzumab plus trastuzumab plus docetaxel for metastatic breast cancer. N Engl J Med 2012; 366: 109-119

[15] Swain SM, Baselga J, Kim SB et al. Pertuzumab, trastuzumab, and docetaxel in HER2-positive metastatic breast cancer. N Engl J Med 2015; 372: 724-734

[16] Gianni L, Pienkowski T, Im YH et al. Efficacy and safety of neoadjuvant pertuzumab and trastuzumab in women with locally advanced, inflammatory, or early HER2-positive breast cancer (NeoSphere): a randomised multicentre, open-label, phase 2 trial. Lancet Oncol 2012; 13: 25-32

[17] Chan A, Delaloge S, Holmes FA et al. Neratinib after trastuzumab-based adjuvant therapy in patients with HER2-positive breast cancer (ExteNET): a multicentre, randomised, double-blind, placebo-controlled, phase 3 trial. Lancet Oncol 2016; 17: 367-377

[18] Martin M, Holmes FA, Ejlertsen B et al. Neratinib after trastuzumab (T)based adjuvant therapy in early-stage HER2+ breast cancer (BC): 5-year analysis of the phase III ExteNET trial. Ann Oncol 2017; 28 (Suppl. 5): v43-v67, Abstr. 1490

[19] Finn RS, Crown JP, Lang I et al. The cyclin-dependent kinase 4/6 inhibitor palbociclib in combination with letrozole versus letrozole alone as firstline treatment of oestrogen receptor-positive, HER2-negative, advanced breast cancer (PALOMA-1/TRIO-18): a randomised phase 2 study. Lancet Oncol 2015; 16: 25-35

[20] Finn RS, Martin M, Rugo HS et al. Palbociclib and letrozole in advanced breast cancer. N Engl J Med 2016; 375: 1925-1936

[21] Turner NC, Ro J, Andre F et al. Palbociclib in hormone-receptor-positive advanced breast cancer. N Engl J Med 2015; 373: 209-219

[22] Hortobagyi GN, Stemmer SM, Burris HA et al. Ribociclib as first-line therapy for HR-positive, advanced breast cancer. N Engl J Med 2016; 375: 1738-1748

[23] Goetz MP, Toi M, Campone M et al. MONARCH 3: abemaciclib as initial therapy for advanced breast cancer. J Clin Oncol 2017. doi:10.1200/ JCO.2017.75.6155

[24] Sledge GW jr., Toi M, Neven P et al. MONARCH 2: abemaciclib in combination with fulvestrant in women with HR+/HER2- advanced breast cancer who had progressed while receiving endocrine therapy. J Clin Oncol 2017; 35: 2875-2884

[25] Sledge GW jr., Toi M, Neven P et al. MONARCH 2: abemaciclib in combination with fulvestrant in patients with $\mathrm{HR}+$ /HER2- advanced breast cancer who progressed on endocrine therapy. J Clin Oncol 2017; 35 (Suppl.): Abstr. 1000

[26] Di Leo A, Toi M, Campone M et al. MONARCH 3: abemaciclib as initial therapy for patients with HR+/HER2-advanced breast cancer. Ann Oncol 2017; 28 (Suppl. 5): v74-v108, Abtr. 2360

[27] Finn RS, Crown J, Lang I et al. Overall survival results from the randomized phase II study of palbociclib $(\mathrm{P})$ in combination with letrozole $(\mathrm{L})$ vs. letrozole alone for frontline treatment of ER+/HER2- advanced breast cancer (PALOMA-1; TRIO-18). J Clin Oncol 2017; 35 (Suppl.): Abstr. 1001 
[28] Hortobagyi GN, Stemmer SM, Burris HA et al. Updated results from MONALEESA-2, a phase 3 trial of first-line ribociclib + letrozole in hormone receptor-positive (HR+), HER2-negative (HER2-), advanced breast cancer (ABC). J Clin Oncol 2017; 35 (Suppl.): Abstr. 1038

[29] Dickler MN, Barry WT, Cirrincione CT et al. Phase III trial evaluating letrozole as first-line endocrine therapy with or without bevacizumab for the treatment of postmenopausal women with hormone receptor-positive advanced-stage breast cancer: CALGB 40503 (Alliance). J Clin Oncol 2016; 34: 2602-2609

[30] Martin M, Loibl S, von Minckwitz G et al. Phase III trial evaluating the addition of bevacizumab to endocrine therapy as first-line treatment for advanced breast cancer: the letrozole/fulvestrant and avastin (LEA) study. J Clin Oncol 2015; 33: 1045-1052

[31] Walsh CS. Two decades beyond BRCA1/2: homologous recombination, hereditary cancer risk and a target for ovarian cancer therapy. Gynecol Oncol 2015; 137: 343-350

[32] Robson M, Im SA, Senkus E et al. Olaparib for metastatic breast cancer in patients with a germline BRCA mutation. N Engl J Med 2017; 377: 523533

[33] Robson ME, Im S-A, Senkus E et al. OlympiAD: Phase III trial of olaparib monotherapy versus chemotherapy for patients (pts) with HER2-negative metastatic breast cancer $(\mathrm{mBC})$ and a germline BRCA mutation (gBRCAm). J Clin Oncol 2017; 35 (Suppl.): Abstr. LBA4

[34] Delaloge S, Conte PF, Im S-A et al. OlympiAD: Further efficacy outcomes in patients with HER2-negative metastatic breast cancer and a germline BRCA mutation receiving olaparib monotherapy vs. standard singleagent chemotherapy treatment of physician's choice. Ann Oncol 2017; 28 (Suppl. 5): v74-v108, Abstr. 243PD

[35] Turner NC, Telli ML, Rugo HS et al. Final results of a phase 2 study of talazoparib (TALA) following platinum or multiple cytotoxic regimens in advanced breast cancer patients (pts) with germline BRCA1/2 mutations (ABRAZO). J Clin Oncol 2017; 35 (Suppl.): Abstr. 1007

[36] Geyer CE, O'Shaughnessy J, Untch M et al. Phase 3 study evaluating efficacy and safety of veliparib $(\mathrm{V})$ plus carboplatin $(\mathrm{Cb})$ or $\mathrm{Cb}$ in combination with standard neoadjuvant chemotherapy (NAC) in patients (pts) with early stage triple-negative breast cancer (TNBC). J Clin Oncol 2017; 35 (Suppl.): Abstr. 520

[37] Couch FJ, Shimelis $\mathrm{H}, \mathrm{Hu} \mathrm{C}$ et al. Associations between cancer predisposition testing panel genes and breast cancer. JAMA Oncol 2017; 3: 1190 1196

[38] Couch FJ, Hart SN, Sharma P et al. Inherited mutations in 17 breast cancer susceptibility genes among a large triple-negative breast cancer cohort unselected for family history of breast cancer. J Clin Oncol 2015; 33: 304-311

[39] Michailidou K, Lindstrom S, Dennis ] et al. Association analysis identifies 65 new breast cancer risk loci. Nature 2017; 551: 92-94

[40] Stevens KN, Vachon CM, Lee AM et al. Common breast cancer susceptibility loci are associated with triple-negative breast cancer. Cancer Res 2011; 71: 6240-6249

[41] Stevens KN, Fredericksen Z, Vachon CM et al. 19p13.1 is a triple-negative-specific breast cancer susceptibility locus. Cancer Res 2012; 72: 1795-1803

[42] Purrington KS, Slager S, Eccles D et al. Genome-wide association study identifies 25 known breast cancer susceptibility loci as risk factors for triple-negative breast cancer. Carcinogenesis 2014; 35: 1012-1019

[43] Milne RL, Kuchenbaecker KB, Michailidou K et al. Identification of ten variants associated with risk of estrogen-receptor-negative breast cancer. Nat Genet 2017. doi:10.1038/ng.3785

[44] Schmid P, Park YH, Muñoz-Couselo E et al. Pembrolizumab (pembro) + chemotherapy (chemo) as neoadjuvant treatment for triple negative breast cancer (TNBC): preliminary results from KEYNOTE-173. J Clin Oncol 2017; 35 (Suppl.): Abstr. 556
[45] Adams A, Loi S, Toppmeyer D et al. Phase 2 study of pembrolizumab as first-line therapy for PD-L1-positive metastatic triple-negative breast cancer (mTNBC): preliminary data from KEYNOTE-086 cohort B. J Clin Oncol 2017; 35 (Suppl.): Abstr. 1088

[46] Stebbing J, Baranau YV, Baryash V et al. Double-blind, randomized phase III study to compare the efficacy and safety of CT-P6, trastuzumab biosimilar candidate versus trastuzumab as neoadjuvant treatment in HER2 positive early breast cancer (EBC). J Clin Oncol 2017; 35: 510

[47] Pivot XB, Bondarenko I, Dvorkin M et al. A randomized, double-blind, phase III study comparing SB3 (trastuzumab biosimilar) with originator trastuzumab in patients treated by neoadjuvant therapy for HER2-positive early breast cancer. J Clin Oncol 2017; 35 (Suppl.): Abstr. 509

[48] Pegram M, Tan-Chiu E, Freyman A et al. A randomized, double-blind study of PF-05280014 (a potential trastuzumab biosimilar) vs. trastuzumab, both in combination with paclitaxel, as first-line treatment for HER2-positive metastatic breast cancer. Ann Oncol 2017; 28 (Suppl. 5): v74-v108, Abstr. 238PD

[49] Lammers PE, Dank M, Masetti R et al. A randomized, double-blind study of PF-05280014 (a potential biosimilar) vs. trastuzumab, both given with docetaxel (D) and carboplatin (C), as neoadjuvant treatment for operable human epidermal growth factor receptor 2-positive (HER21) breast cancer. Ann Oncol 2017; 28 (Suppl. 5): v43-v67, Abstr. 154PD

[50] Pivot X, Bondarenko IM, Nowecki Z et al. One-year safety, immunogenicity, and survival results from a phase III study comparing SB3 (a proposed trastuzumab biosimilar) and originator trastuzumab in HER2-positive early breast cancer treated with neoadjuvant-adjuvant treatment. Ann Oncol 2017; 28 (Suppl. 5): v43-v67, 153PD

[51] Esteva F], Baranau Y, Baryash V et al. Double-blind, randomized phase III study to compare the efficacy and safety of trastuzumab and its biosimilar candidate CT-P6 in HER2 positive early breast cancer (EBC). Ann Oncol 2017; 28 (Suppl. 5): v43-v67, 152PD

[52] von Minckwitz G, Ponomarova O, Morales S et al. Efficacy and safety of biosimilar ABP 980 compared with trastuzumab in HER2 positive early breast cancer. Ann Oncol 2017; 28 (Suppl. 5): v43-v67, Abstr. 151PD

[53] Hadji P, Blettner M, Harbeck N et al. The Patient's Anastrozole Compliance to Therapy (PACT) Program: a randomized, in-practice study on the impact of a standardized information program on persistence and compliance to adjuvant endocrine therapy in postmenopausal women with early breast cancer. Ann Oncol 2013; 24: 1505-1512

[54] Hadji P, Jackisch C, Bolten W et al. COMPliance and Arthralgia in Clinical Therapy: the COMPACT trial, assessing the incidence of arthralgia, and compliance within the first year of adjuvant anastrozole therapy. Ann Oncol 2014; 25: 372-377

[55] Nabieva N, Kellner S, Fehm T et al. Patient and tumor characteristics and their influence on early therapy persistence with letrozole in postmenopausal patients with early breast cancer. Ann Oncol 2017. doi:10.1093/ annonc/mdx630

[56] Basch EM, Deal AM, Dueck AC et al. Overall survival results of a randomized trial assessing patient-reported outcomes for symptom monitoring during routine cancer treatment. J Clin Oncol 2017; 35 (Suppl.): Abstr. LBA2

[57] Henry NL, Azzouz F, Desta Z et al. Predictors of aromatase inhibitor discontinuation as a result of treatment-emergent symptoms in early-stage breast cancer. J Clin Oncol 2012; 30: 936-942

[58] Chirgwin JH, Giobbie-Hurder A, Coates AS et al. Treatment adherence and its impact on disease-free survival in the Breast International Group 1-98 Trial of Tamoxifen and Letrozole, Alone and in Sequence. J Clin Oncol 2016; 34: 2452-2459

[59] ClinicalTrials.gov. Impact of eHealth-support on quality of life in metastatic breast cancer patients treated with palbociclib and endocrine therapy (PRECYCLE). 2017. Online: https://clinicaltrials.gov/ct2/show/ NCT03220178; Stand: 03.11.2017 
[60] Hartkopf AD, Graf J, Simoes E et al. Electronic-based patient-reported outcomes: willingness, needs, and barriers in adjuvant and metastatic breast cancer patients. JMIR Cancer 2017; 3: e11

[61] Wallwiener M, Heindl F, Brucker SY et al. Implementation and feasibility of electronic Patient-Reported Outcome (ePRO) data entry in the PRAEGNANT real-time advanced and metastatic breast cancer registry. Geburtsh Frauenheilk 2017; 77: 870-878

[62] Wallwiener M, Matthies L, Simoes E et al. Reliability of an e-PRO tool of EORTC QLQ-C30 for measurement of health-related quality of life in patients with breast cancer: prospective randomized trial. J Med Internet Res 2017; 19: e322
[63] Fasching PA, Brucker SY, Fehm TN et al. Biomarkers in patients with metastatic breast cancer and the PRAEGNANT study network. Geburtsh Frauenheilk 2015; 75: 41-50

[64] Fasching PA, Lux MP, Häberle L et al. SERAPHINA - Safety, Efficacy and patient Reported outcomes of Advanced breast cancer Patients: tHerapy management wlth NAb-paclitaxel in daily routine (a non-interventional study). Senologie 2016; 13: A24A 